\title{
Accurate seabed modeling using finite difference methods
}

\author{
Gang Yao ${ }^{1}$ (D) Nuno Vieira da Silva ${ }^{1} \cdot$ Henry Alexander Debens ${ }^{1} \cdot$ Di Wu$^{2}$
}

Received: 29 January 2017 / Accepted: 2 November 2017 / Published online: 18 November 2017

(C) The Author(s) 2017. This article is an open access publication

\begin{abstract}
Finite difference is the most widely used method for seismic wavefield modeling. However, most finitedifference implementations discretize the Earth model over a fixed grid interval. This can lead to irregular model geometries being represented by 'staircase' discretization, and potentially causes mispositioning of interfaces within the media. This misrepresentation is a major disadvantage to finite difference methods, especially if there exist strong and sharp contrasts in the physical properties along an interface. The discretization of undulated seabed bathymetry is a common example of such misrepresentation of the physical properties in finite-difference grids, as the seabed is often a particularly sharp interface owing to the rapid and considerable change in material properties between fluid seawater and solid rock. There are two issues typically involved with seabed modeling using finite difference methods: firstly, the travel times of reflections from the seabed are inaccurate as a consequence of its spatial mispositioning; secondly, artificial diffractions are generated by the staircase representation of dipping seabed bathymetry. In this paper, we propose a new method that provides a solution to these two issues by positioning sharp interfaces at fractional grid locations. To achieve this, the velocity
\end{abstract}

Gang Yao

g.yao@imperial.ac.uk

1 Department of Earth Science and Engineering, Imperial College London, London SW7 2BP, UK

2 The Unconventional Natural Gas Institute, China University of Petroleum (Beijing), Beijing 102249, China model is first sampled in a model grid that allows the center of the seabed to be positioned at grid points, before being interpolated vertically onto a regular modeling grid using the windowed sinc function. This procedure allows undulated seabed bathymetry to be represented with improved accuracy during modeling. Numerical tests demonstrate that this method generates reflections with accurate travel times and effectively suppresses artificial diffractions.

Keywords Finite difference $\cdot$ Staircase $\cdot$ Seabed bathymetry $\cdot$ Integer grid $\cdot$ Fractional grid $\cdot$ Sinc interpolation

\section{Introduction}

Seismic wavefield modeling is a key component of seismic imaging, e.g., reverse-time migration $[3,27,33,49,50,52$, 53, 60], and inversion, e.g., full-waveform inversion [36, 37, 43, 48]. Due to the heterogeneity of the Earth, the simulation of seismic wavefields is achieved most commonly using numerical methods, such as the finite-element method $[8,30,35]$, the finite-difference method [1, 25, 44, 45], spectral-element method [23, 24], or discontinuous Galerkin $[6,7,11,15,21]$. The most widely used is the finitedifference method, which uses finite differences to calculate the partial derivatives of wave equations numerically. Finite difference's enduring popularity derives from its simplicity of implementation and computational efficiency, yet a high degree of accuracy. However, the representation of model continua by discrete grids will cause finite difference to suffer from numerical dispersion; optimized stencils for the finite-difference method have been introduced with the aim of mitigating this dispersion $[9,28,47,51,58]$. 
In the majority of finite-difference applications, fixed regular grids are used to represent the seismic properties of the subsurface, such as acoustic velocity or density. This approach has two undesirable consequences when material interfaces with irregular geometries do not align well to the sampling grid. Firstly, the position of these interfaces will be misrepresented by the discretized model, resulting in the incorrect travel times of propagating energy [42]. Secondly, as a consequence of being sampled by fixed regular grids, irregularly shaped interfaces will typically need to be represented in a "staircase" fashion. It is preferable to avoid this kind of staircase representation during discretization, as their corners will commonly generate a series of artificial diffractions. A commonly seen example of a staircase discretization is when modeling through a dipping seabed using finite difference schemes. The effects here are often most conspicuous, as the seabed is a sharp interface owing to the fast transition in material properties between fluid seawater and solid rock.

Since seismic wavefields contain band-limited frequencies, they are insensitive to high-frequency components of the sharp interfaces. Thus, a means to discretize sharp interfaces is to smooth the interfaces before discretization [5]. However, this method can cause inaccurate reflection structure [13].

The simplest method to achieve a more accurate representation of irregular interfaces is to use finer-grid spacing. This is because when the time and space steps are close to zero, the finite-difference solution converges to the exact solution. A grid with at least 30 points per shortest wavelength can deliver reasonably accurate simulation [46]. However, the cost of computation will increase dramatically as the number of grid points increases, as well as with the associated increase in the number of simulation time steps required. For instance, if the grid size is reduced by half, then the computational cost for 3D modeling increases by 16 times as a result.

Alternatively, numerical methods that can account for the irregular nature of interfaces can be used. For example, the finite-element method $[8,30,35]$ can approximate complex geometries and topographies by discretizing the models using triangular or tetrahedral cells. Other similar methods, for example, the discontinuous Galerkin $[6,7,11,15,21]$, the finite-volume method $[10,57,59]$, and the boundaryelement method [31,32], can also provide a measure of flexibility towards rugged interfaces by employing irregular grids. However, compared with finite-difference methods, these alternatives are almost always more computationally expensive and generally more complicated to implement.

A compromise between complexity of implementation, computational load, and accuracy can be obtained via the combination of finite-difference methods and coordinate transformations. To achieve this, the continuous model is first sampled along any problematic interfaces to form a curved grid; the curved grid is then mapped to an orthogonal grid via coordinate transform. There are two widely used methods: the curvilinear coordinate method $[16,17]$ and the body-fitted grid method $[22,38,55]$. Both these methods are commonly used to model irregular topography of the free surface. However, both have the limitation that they skew the model grid, whereby spurious wavefield features will be generated at locations where the angle between grid vertices is small or the cell dimensions change rapidly [38]. The coordinate mapping can also lead to small cells in the original coordinates resulting in small time step to ensure stable modeling [39]. In addition, both methods also involve a significant computational overhead. For instance, in the case of the acoustic wave equation, the body-fitted grid method can be three times as computationally expensive as the standard finite-difference method [38].

Since seismic wavefields and the product of the model parameters, e.g., velocity, and the spatial derivatives of wavefields are continuous around the interface, an alternative way to deal with the interface located between grid points is to impose the continuity conditions of wavefields around the interface into a finite-difference scheme with regular grids. This type of methods is called "immersed interface method" [26]. It was originally developed with second-order accuracy $[26,56]$ and then further extended to the fourth-order accuracy for both acoustic and elastic wave equations [54]. The immersed interface method requires knowledge about the jumps in the model parameters and their one-sided derivatives [42]. In addition, it is relatively difficult to derive and implement, especially for the accuracy of order higher than four. The similar methods called "immersed boundary method" are also used to deal with an irregular topography of the free surface $[19,29]$.

Dealing with the discontinuous interfaces, in fact, can be classified as a homogenization problem, which produces an equivalent medium by averaging to replace the heterogeneous media under the assumption of the wavelength much larger than the scale of the heterogeneity [12]. Based on this theory, smoothing the grid points around the interface can give second-order accuracy to approximate the boundary conditions of the interface [5]. Muir et al. [34] applied the S-M calculus [40], which is a general formulation of Backus averaging [2], to compute the equivalent value of elastic parameters for the grid points near the discontinuous interfaces [34]. Their results demonstrated that the averaging method can effectively suppress the spurious diffractions [34]. The idea of averaging for dealing with discontinuous interfaces has also been applied with the finite-element methods with a lumped mass matrix, which leads to an effective interface-smoothing scheme by summing the rows of the mass matrix for constant-density acoustic media [41]. 
In this paper, we propose a novel approach to accurately model undulated seabed bathymetry by positioning them on a fractional grid. Numerical examples demonstrate that this method generates reflection events with correct travel times and avoids artificial diffractions contaminating the wavefield.

\section{Theory}

To demonstrate the principles behind our method, we consider the constant-density acoustic wave equation, given by

$\frac{\partial^{2} p}{\partial t^{2}}-v^{2} \nabla^{2} p=s(t)$

where $p$ is the pressure wavefield, $s$ is the source term, and $v$ is acoustic velocity. Although our derivation is for only acoustic velocity, other model properties (such as density) can be generated in the same manner. Wave equations assume that the wavefield is continuous and that interfaces of different materials are represented by the variation of velocity. As a result, scattered waves can be thought of as a response of wavefield "stretching" from the multiplication of the spatially varying $v^{2}$ and $\nabla^{2} p$ in Eq. 1 . As such, the locations where scattered energy is generated correspond to positions where the velocity changes along the path of wave propagation. If Eq. 1 is solved analytically then scattered arrivals can be computed accurately. Analytical solutions are only achievable for very simple Earth models; however, when considering a heterogeneous subsurface, numerical methods are necessary for the solution of Eq. 1. The finite-difference method requires the model properties to be discretized along the dimensions of the model. Usually, the discretization along the vertical direction starts from the model surface over a fixed interval. This discrete model generated with this standard way is referred to as an integer grid model (IGM) in this paper. Often this results in interfaces between media being represented poorly by the discretized model when the interfaces are not aligned with the finite-difference grid.

In most marine environments worldwide, at the seabed, there can be found a rapid transition in material properties between fluid seawater and solid rock. This manifests itself in most velocity models as a thin interface between strongly contrasting media. If the interface is much thinner than the grid size of the finite difference scheme, the modeling with IGM cannot capture the interface position accurately. As a result, the travel time of reflections is inaccurate. Symes and Vdovina [42] proved that misposition of a thin or discontinuous interface leads to a first-order time-shift error proportional to the distance between the interface and the computational grid. Rather than using a very fine grid to more accurately capture the interface, we can capture the interface accurately by placing a grid point on the center of the interface, where the model stretches the wavefields to originate scattered waves.

This analysis points to a simple approach for accommodating accurately any sharp interfaces within velocity models for wavefield simulation. Firstly, the model should be discretized in a way that places a grid point at the center of the target interface(s). Doing this will likely result in the model grids no longer overlapping the wavefield grid used for modeling. To avoid this, secondly, the discretized model can be interpolated back onto the modeling grid using the sinc function. This process can be expressed as

$\tilde{v}(z)=\sum_{i=-\infty}^{\infty} v(z+\Delta s+i \Delta z) \operatorname{sinc}(\Delta s+i \Delta z)$,

where $\Delta z$ is the grid spacing, $\Delta s$ is the shift between the model grid generated in the first step and the wavefield grid, $v$ is the discretized velocity model in the first step, and $\tilde{v}$ is the interpolated velocity model. As a result, the wave equation of Eq. (1) becomes

$$
\frac{\partial^{2} p}{\partial t^{2}}-\tilde{v}^{2} \nabla^{2} p=s(t) .
$$

For the purposes of wavefield simulation, the interpolated model $\tilde{v}(z)$ is equivalent to the model that positioned the target interface on a fractional grid. We therefore refer to the interpolated model as a fractional-grid model (FGM). As can be seen from Eq. 3, FGMs are compatible with existing finitedifference algorithms. This technique therefore does not require modifications to the existing finite-difference codes or have any salient computational overburden associated.

Figure 1 shows an example of comparison between the IGM method and the FGM method for a sharp interface. As can be seen from Fig. 1a, both interpolated models from the IGM and the FGM contain oscillations around the seabed, and as such, neither is identical to the original model. Nonetheless, the FGM represented by the blue curve crosses precisely the central sample of the true seabed. As we know, the process that waves propagate through a heterogeneous region of velocity to generate scattered waves can be interpreted as a convolution process, in which the scattered waves are formulated as a convolution of source wavelet and the reflectivity of the velocity variation [4]. As a result, the missed wavenumber components, normally the high wavenumber, in the model or the wavelet do not appear in the scattered waves. We, therefore, should focus on the accuracy of the low-wavenumber components of the discrete models. If filtering the original model and the two resampled models with a band-limited Gaussian wavelet, we can see that the filtered FGM rather than the filtered IGM 

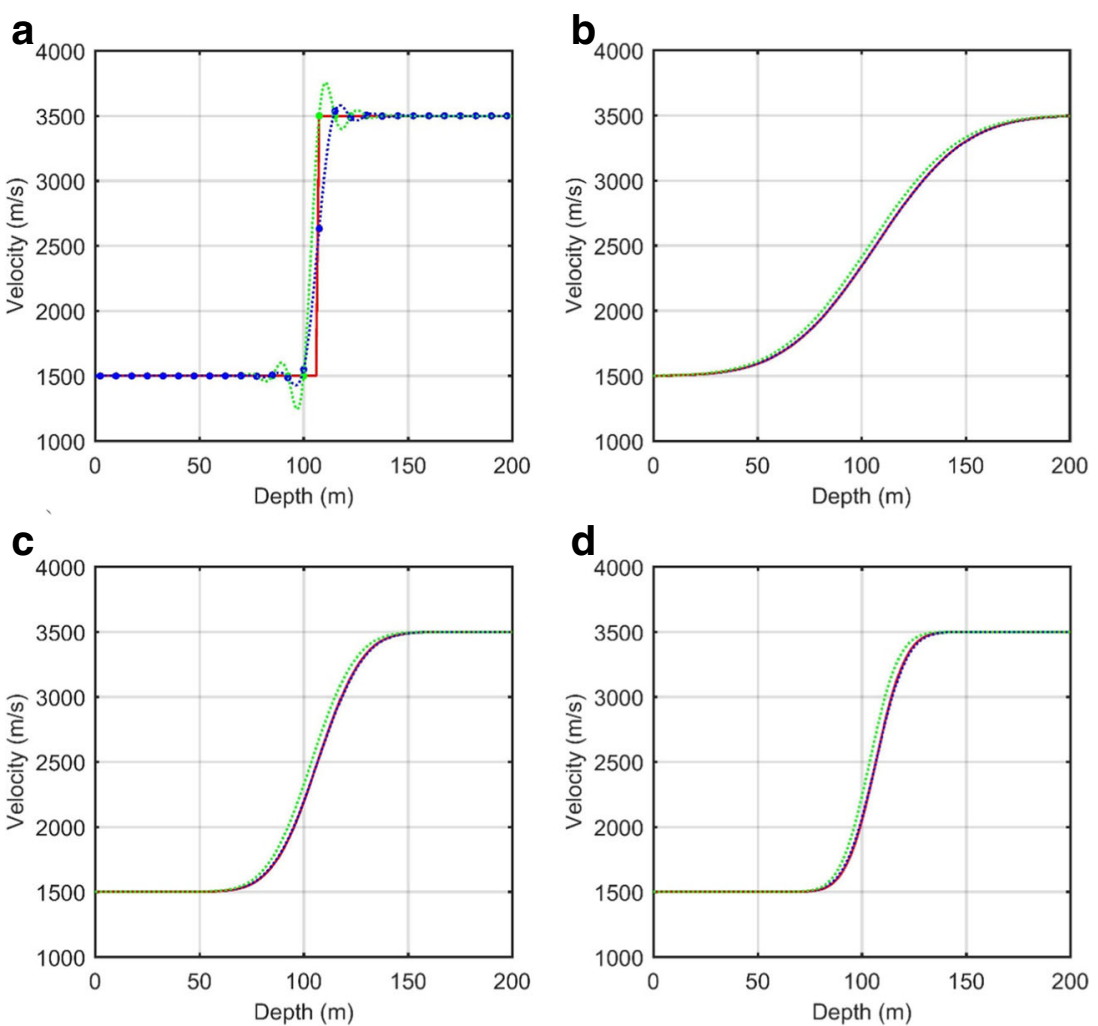

Fig. 1 Resampling, interpolation, and low-pass filtering of a twolayer 1D model. The red curve in (a) represents the true model, which is discretized over an interval of $0.75 \mathrm{~m}$. The seabed here is represented by three contiguous samples, and the velocities of which are 1500 , 2500 , and $3500 \mathrm{~m} / \mathrm{s}$. The center of the seabed is at a depth of 106.75 $\mathrm{m}$. The blue and green circles in (a) represent the model elements resampled over an interval of $7.5 \mathrm{~m}$ with the FGM and IGM methods, respectively. The dotted blue and green curves represent the sinc

matches the filtered original model. This means the FGM is effectively equivalent to the original model when used for wavefield simulation.

\section{Implementation}

\subsection{Sinc function truncation using Kaiser windows}

Equation 2 defines a convolution between the resampled model $v$ and the sinc function, summed over an infinite number of terms. Performing such an operation is not computationally feasible. However, the wavefield is band limited. In order to circumvent numerical dispersion in the modeling with the finite-difference method, the maximum wavenumber of the wavefield is much smaller than the Nyquist wavenumber for the chosen sample interval. As a result, the convolution will usually only need to be accurate for a small range of wavenumbers; this can be achieved using a truncated sinc function. One option is to shape the

interpolation of the resampled models given by the blue and green circles, respectively. In (b), the three models of (a) have been lowpass filtered using a Gaussian function with a central wavenumber of $0.0067 \mathrm{~m}^{-1}$ (corresponding to a $10-\mathrm{Hz}$ Gaussian wavelet traveling at $1500 \mathrm{~m} / \mathrm{s}$ ). c, d Equivalent panels to (b), but in the case of model filtering using functions that correspond to 20 and $30 \mathrm{~Hz}$ Gaussian wavelets, respectively.

sinc function using Kaiser windows [20], which are given by

$W(x)=\left\{\begin{array}{lrr}\frac{I_{0}(b \sqrt{1-x / r})}{I_{0}(b)} & \text { for } & -r \leq x \leq r \\ 0 & \text { otherwise, }\end{array}\right.$

where $r$ is the window half-width and $I_{0}$ is the zeroth-order modified Bessel function of the first kind. The window's properties are dictated by the combination of $r$ and the control parameter $b$, where Hicks [18] provides an analysis into selecting optimal values for these. Two common options are $b=6.31$ for $r=4$ and $b=12.53$ for $r=8$ (Fig. 2). In this paper, we choose the second option of $r=8$, which can be accurate till up to $60 \%$ of the Nyquist frequency (Fig. 2b). Equation 2 now becomes

$\tilde{v}(z)=\sum_{i=-r}^{r} v(z+\Delta s+i \Delta z) W(\Delta s+i \Delta z) \operatorname{sinc}(\Delta s+i \Delta z)$. 
Fig. 2 a Kaiser-windowed sinc functions for $r=8$ and $b=12.53$. b The spectral amplitude of the

Kaiser-windowed sinc function shown in (a). The dashed blue line indicates the spectral amplitude of the unwindowed sinc function. The horizontal axis is the frequency normalized by the sampling frequency; 0.5 corresponds to the Nyquist frequency a

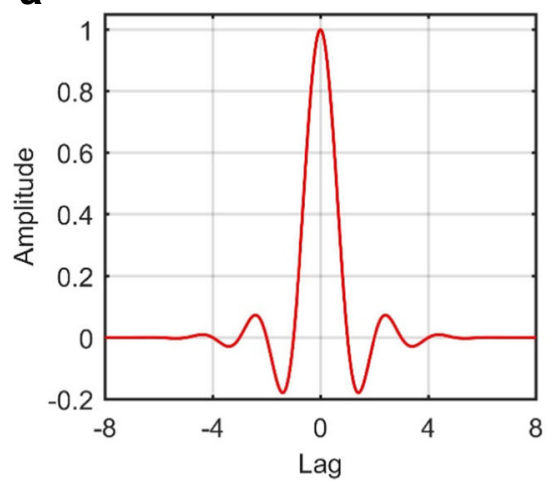

b

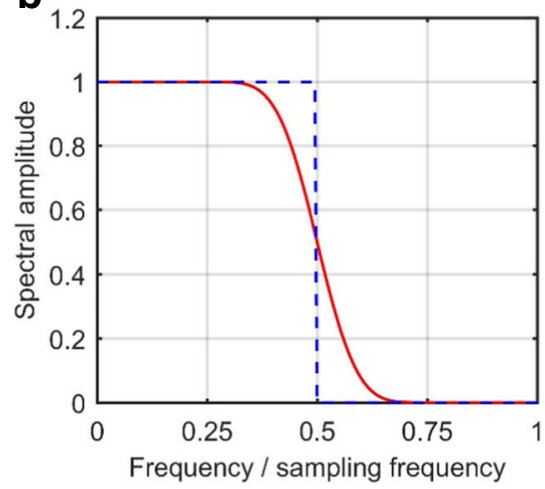

\subsection{Algorithm description}

Based on the theory presented in the previous section, accurate modeling of the seabed can be achieved in two steps:

\section{Discretise the model}

The wavefield $p$ is discretized in space using a uniform spacing. This is illustrated by the black squares in Fig. 3. The seabed interface in this example is located at a fractional position between wavefield grid points and is represented by the red line in Fig. 3. For the seabed to be modeled accurately, the model needs to be rediscretized such that

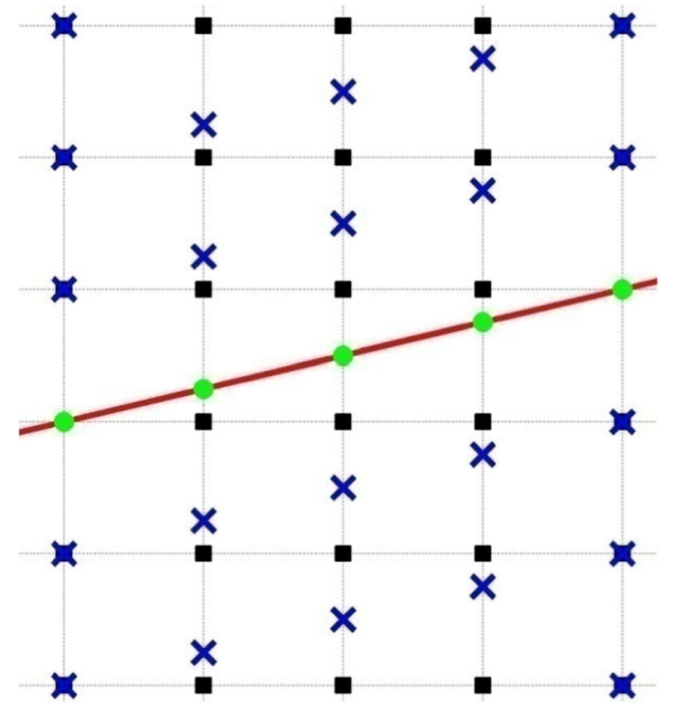

Fig. 3 Schematic illustration of a sharp seabed interface positioned on a fractional grid: the dashed black lines and squares represent the modeling grid for the wavefield; the dipping red line represents the seabed interface; and the blue crosses and green dots represent the model grid. The velocity model has been discretized using the same spatial sampling rate as the wavefield grid and is colocated horizontally with the modeling grid but with each grid point shifted vertically relative to the corresponding wavefield grid point. This shift to the model grid ensures that there exist grid points positioned exactly along the interface at each horizontal interval there is a grid point positioned exactly at the interface. In Fig. 3, these grid points are represented by green dots. Since the sharp transition zone of the seabed may be even thinner than one cell, which is a discontinuous interface effectively, the property values at the interface (green dots) can be replaced with the average value of seawater and the underlying material. According to the homogenization theory, the arithmetic average should be used for density while the harmonic average should be used for the bulk modulus [12]. This procedure can be expressed as

$\bar{\rho}\left(z_{i}\right)=\frac{1}{\Delta z} \int_{z_{i}-0.5 \Delta z}^{z_{i}+0.5 \Delta z} \rho(z) \mathrm{d} z$,

and

$\frac{1}{\bar{k}\left(z_{i}\right)}=\frac{1}{\Delta z} \int_{z_{i}-0.5 \Delta z}^{z_{i}+0.5 \Delta z} \frac{1}{k(z)} \mathrm{d} z$,

where $\rho(z)$ and $k(z)$ are the density and the bulk modulus around the interface (the center of the transition zone), respectively, $\bar{\rho}\left(z_{i}\right)$ and $\bar{k}\left(z_{i}\right)$ are the arithmetic average density and the harmonic average bulk modulus, respectively, $z_{i}$ is the depth of the interface and $\Delta z$ is the vertical grid size. After the average density and bulk modulus are computed, the average velocity can be calculated accordingly. The advantage of computing the average velocity at a discontinuous interface with the homogenization theory is that it can produce an accurate travel time for the reflections generated at the interface; however, a minor drawback is the reflectivity of the interface becomes slightly weaker than its original one since the sharpness is degraded by adding a transition point.

The other grid points of this new discretization are distributed evenly across the remaining model. It can be seen in Fig. 3 that each column of the original model grid has undergone a vertical shift with respect to the wavefield grid.

2. Interpolate the resampled velocity model onto the wavefield grid using a windowed sinc function 
Following step 1, the model grid-represented by the blue crosses and green dots in Fig. 3-is no longer aligned with the wavefield grid, represented by the black squares. This can be rectified by interpolation of the velocity model onto the wavefield grid using Eq. 4. By doing this, Eq. 1 can be time stepped numerically following

$$
\begin{gathered}
p(x, z, t+\Delta t)=2 p(x, z, t)-p(x, z, t-\Delta t) \\
+\Delta t^{2} \tilde{v}^{2}(x, z) \nabla^{2} p(x, z, t)+s(t)
\end{gathered},
$$

where $\Delta t$ is the time-stepping interval.

\section{Examples}

\subsection{Accurate travel times}

The first example uses a simple two-layer model (Fig. 4) to demonstrate that FGMs can produce accurate travel times. The upper layer contains seawater with an acoustic velocity of $1500 \mathrm{~m} / \mathrm{s}$ and a density of $1000 \mathrm{~kg} / \mathrm{m}^{3}$, while the lower layer is composed of sedimentary rock with an acoustic velocity of $3500 \mathrm{~m} / \mathrm{s}$ and a density of $2000 \mathrm{~kg} / \mathrm{m}^{3}$. The model is discretized over grid points spaced $7.5 \mathrm{~m}$ apart in each dimension. The horizontal seabed interface is located at a depth of $1500 \mathrm{~m}$ and has a single velocity of $1658 \mathrm{~m} / \mathrm{s}$ and a density of $1500 \mathrm{~kg} / \mathrm{m}^{3}$, which are the average of the two layers and computed with Eqs. 5 and 6. The source is

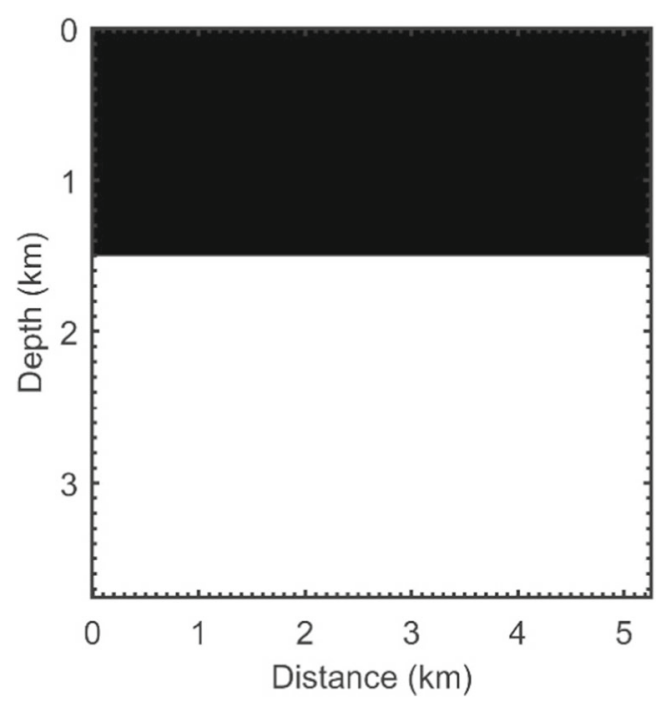

Fig. 4 The schematic diagram of a two-layer acoustic model: the top layer contains seawater with a velocity of $1500 \mathrm{~m} / \mathrm{s}$ and a density of $1000 \mathrm{~kg} / \mathrm{m}^{3}$ while the bottom layer contains rock with a velocity of $3500 \mathrm{~m} / \mathrm{s}$ and a density of $2000 \mathrm{~kg} / \mathrm{m}^{3}$. According to the homogenization theory, the interface at a depth of $1500 \mathrm{~m}$ has a velocity of 1658 $\mathrm{m} / \mathrm{s}$ and a density of $1500 \mathrm{~kg} / \mathrm{m}^{3}$ a Ricker wavelet with a dominant frequency of $10 \mathrm{~Hz}$ and a sample rate of $1 \mathrm{~ms}$. A single synthetic shot is generated at the top surface and a horizontal position of $2625 \mathrm{~m}$.

If the model is sampled vertically from the top surface in the standard manner, then the seabed is exactly coincident with a row of grid points. This is an IGM. For comparison, we also generate FGMs with vertical shifts of $0,-0.3,-0.5$, and -0.7 grid points, such that the interface is moved incrementally towards the top surface. However, the IGM does not change when moving the interface. Velocity and density profiles through the seabed interface from each model are shown in Fig. 5. Finite-difference modeling using the IGM produces the shot gather displayed in Fig. 6a, while Fig. $6 \mathrm{~b}$ displays an equivalent gather produced using the FGM where the interface has been shifted up by half a cell. These two records appear very similar, and require closer inspection to expose their differences. The central trace of each gather, along with traces modeled using the other three FGMs of Fig. 5, is shown in Fig. 7. Compared with the IGM trace, the FGM traces can be seen to contain time shifts, which are caused by the repositioned seabed interface in the FGMs. The time shifts for the $-0.3,-0.5$, and -0.7 grid point FGMs are $-3,-5$, and $-7 \mathrm{~ms}$, respectively. These time shifts therefore match exactly the expected values. In the case where the FGM shift is $0 \mathrm{~m}$, the modeled trace (blue) matches exactly the IGM trace (red) in Fig. 7b. These results demonstrate empirically that FGMs can accurately capture the seabed's kinematic properties regardless of whether the interface is coincident with the modeling grid.

This example exposes a minor drawback to the FGM method: it can produce small errors in amplitude. These errors are best observed in Fig. 7b, which demonstrates that the reflections produced using the depth-shifted FGMs contain different amplitudes relative to the non-shifted FGM, up to a maximum difference of $0.35 \%$. In real-world applications such as full-waveform inversion, these minimal errors will not result in any adverse effects to the inversion result.

\subsection{Suppressed artificial diffractions}

If the interface of a dipping seabed does not align with the modeling grid, then it will require being represented by a series of staircase structures when discretized using the IGM method. To demonstrate this, we use the same model as the previous example, but the centermost section of the seabed is now dipping gently at $5.71^{\circ}$ to the left (Fig. 8). After discretization with the IGM method, the dipping seabed is characterized by a strong staircase signature, as highlighted in Fig. 8a, c. Using the same acquisition geometry as in the first example, finite-difference modeling using this IGM produces the synthetic record shown in Fig. 9a. This gather 
a

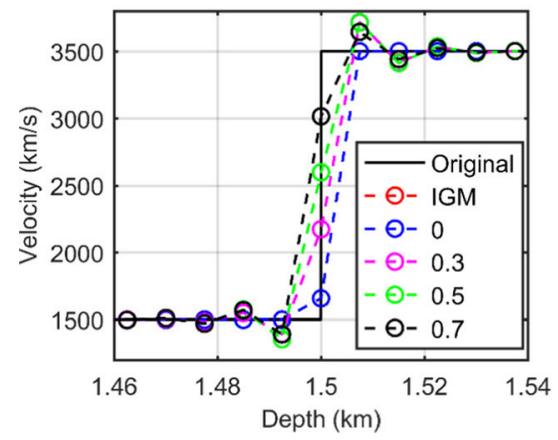

Fig. 5 Velocity (a) and density (b) vertical profiles through the IGM and four FGMs, where each FGM positions the seabed interface slightly differently relative to the IGM. The solid black curve represents the original model (Fig. 4). The dashed blue, magenta, green, and black curves represent the various FGMs, within which the interface

contains a noticeable sequence of hyperbolic diffractions that follow the primary seabed reflection, which are a result of the staircase corners' scattering nature. The true model, however, is known to contain a smooth seabed interface throughout, so these diffraction events are noise.

By systematically shifting the model grid vertically by a small distance, the seabed interface can be aligned with the grid. Consequently, an FGM can be generated using these shifts (Fig. 8,d). Finite-difference modeling using this FGM produces the record shown in Fig. 9b. Comparing Fig. 9a, b, it can be seen that using the FGM avoids completely the spurious diffractions produced when using the IGM. Figure 10 compares the central trace of the shot gathers in Fig. 9, as indicated by the red dotted line through each gather. The trace produced using the IGM (red line) contains strong oscillations after the main reflection event; however, the trace obtained using the FGM (blue line) is free of such oscillations. Figure 11 displays a snapshot of the wavefield at $1.5 \mathrm{~s}$ when using each of the models in Fig. 8 for finitedifference modeling. It is clear from these snapshots that the spurious diffractions are not present when using the FGM. b

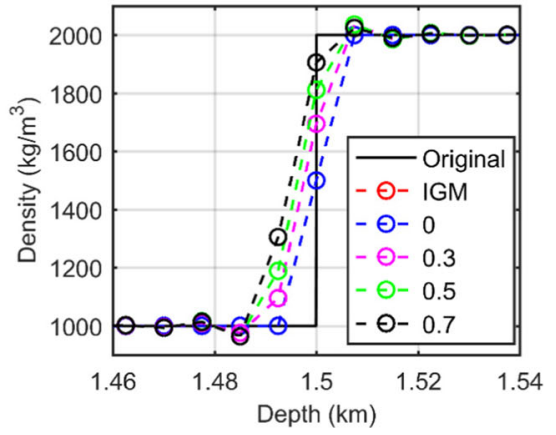

has been shifted upwards by $0,-0.3,-0.5$, and -0.7 grid points, respectively. The IGM discretization of the original model is represented by the dashed red curve, which in this example is overlapped exactly by the blue curve. The circles represent the discretized samples

\subsection{Suppressed artificial diffractions—efficacy with dip angle}

The previous example demonstrated that the use of FGMs during finite-difference modeling can avoid the spurious diffractions that occur as a result of dipping interfaces being represented in a staircase fashion. In this example, we examine the limit of interface dip angle for which FGMs can maintain successful suppression of diffractions. To go about this, we generate a suite of 91 two-layer acoustic velocity and density models, each of which contains a different angle of interface dip between the two layers. These angles range monotonically between $0^{\circ}$ and $90^{\circ}$ over an increment of $1^{\circ}$ (Fig. 12a). The upper layer contains seawater with a constant velocity of $1500 \mathrm{~m} / \mathrm{s}$ and a constant density of $1000 \mathrm{~kg} / \mathrm{m}^{3}$, while the lower layer represents soft sedimentary rock with a velocity of $1800 \mathrm{~m} / \mathrm{s}$ and a constant density of $1200 \mathrm{~kg} / \mathrm{m}^{3}$. Each model is discretized using the FGM method over a $7.5 \times 7.5 \mathrm{~m}$ grid size, and a single source and receiver are located at the center of the model. In every model, the minimum source-interface distance is
Fig. 6 Synthetic shot gathers produced using a the IGM, where the seabed interface is located exactly at an integer grid position (at a depth of $1500 \mathrm{~m}$ ) and $\mathbf{b}$ the FGM, where the seabed interface has been shifted upwards by half a cell relative to the IGM
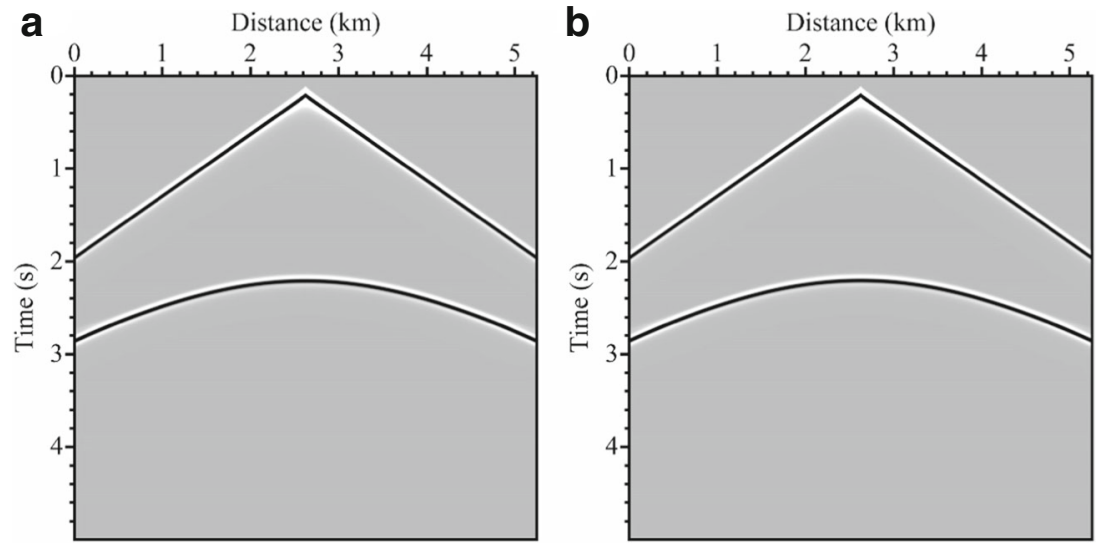


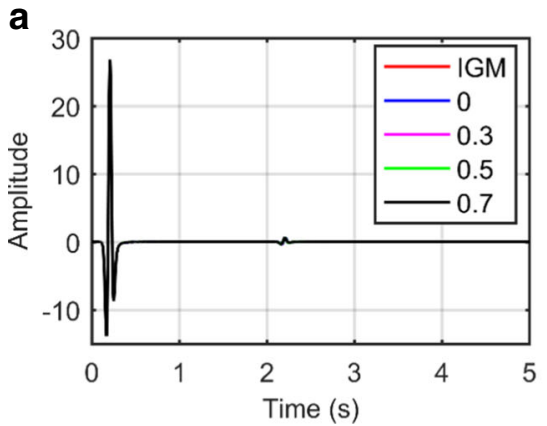

Fig. 7 Traces taken at a horizontal distance of $2625 \mathrm{~m}$ from several synthetic shot gathers generated using different velocity models: a the complete record length and $\mathbf{b}$ an enlarged view of the reflection events generated by the seabed interface in (a). Note that the horizontal position is coincident with the shot location. The red trace was generated using the IGM, within which the interface is located exactly at an integer grid position (at a depth of $1500 \mathrm{~m}$ ); this trace is in both panels

$1.5 \mathrm{~km}$; as a result, the first back-scattered arrival occurs simultaneously for all models. The source signature is a Ricker wavelet with a dominant frequency of $10 \mathrm{~Hz}$ and a maximum spectral content of $25 \mathrm{~Hz}$, corresponding to eight samples per wavelength in the water layer. Figure $12 \mathrm{~b}$ shows the trace recorded from each model collected into a single gather, from which the direct arrival has been subtracted. Figure 12c shows the same gather as Fig. 12b but recorded b

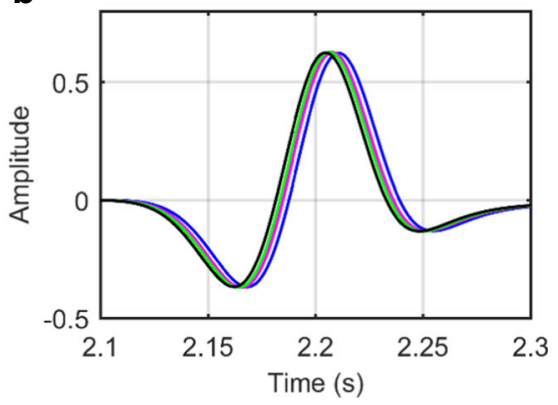

overlapped exactly by the blue trace. The blue, magenta, green, and black traces were generated using the FGMs, within which the interface has been shifted upwards by $0,-0.3,-0.5$, and -0.7 grid points, respectively, relative to the IGM. The magenta, green, and black traces contain $-3,-5$, and $-7 \mathrm{~ms}$ shifts, respectively, relative to the blue and red traces. The time shifts observed match the theoretically expected ones

using a Ricker source wavelet with a dominant frequency of $20 \mathrm{~Hz}$. In this case, the highest frequency propagated is around $50 \mathrm{~Hz}$, which corresponds to four samples per wavelength in the water layer. By inspecting Fig. 12b, c, it is apparent that there is significant noise following the primary reflection event when the interface is dipping steeply, suggesting that the FGM method is no longer accurately representing the true interface.
Fig. 8 Discretization of a simple two-layer model containing a dipping seabed. The model dimensions are the same as in Fig. 4, but in this case, the interface between the two layers has a section dipping constantly at $5.71^{\circ}$ between 0.75 and 4.25 $\mathrm{km}$ horizontal distance. The left horizontal section is positioned at $1.5 \mathrm{~km}$ depth, while the right horizontal section is at $1.125 \mathrm{~km}$ depth. Discretizing the model onto differently positioned 7.5 $\times 7.5 \mathrm{~m}$ grids results in the IGMs of a velocity and $\mathbf{c}$ density and the FGMs of $\mathbf{b}$ velocity and $\mathbf{d}$ density. The red line in both subfigures indicates the true position of the seabed interface. The interfaces in (a) and (c) are staggered into a series of sudden steps in model properties, one of which appears every ten columns, whereas the interfaces in (b) and (d) are represented by a smoother transition in model properties

\section{a}

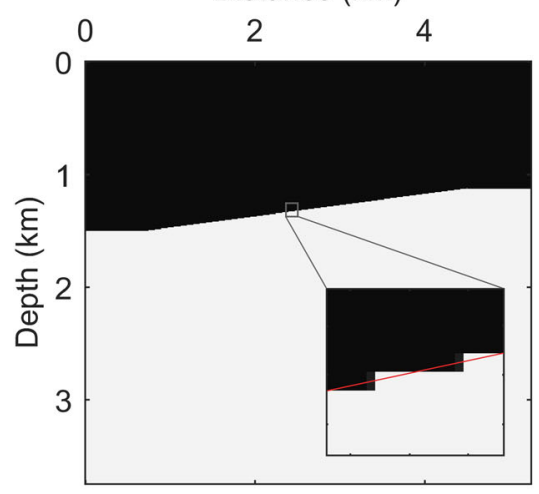

$$
\text { C }
$$

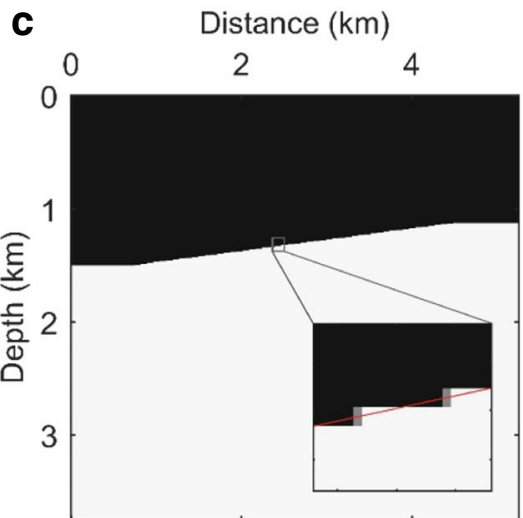

b

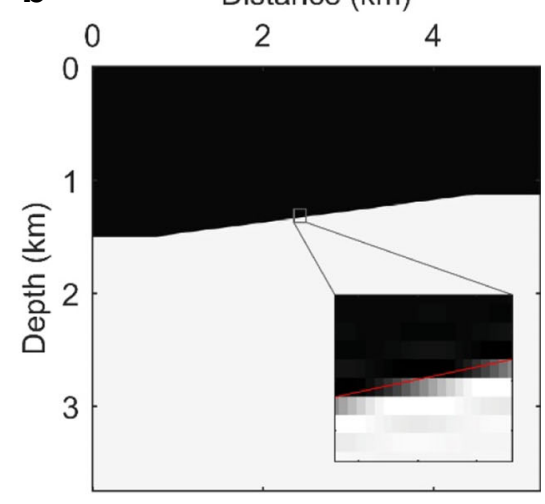

d

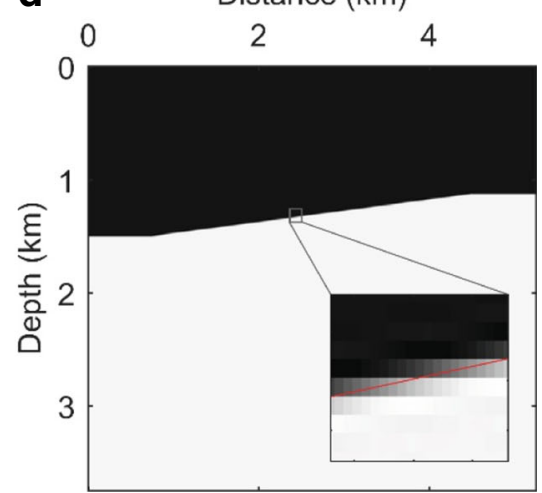




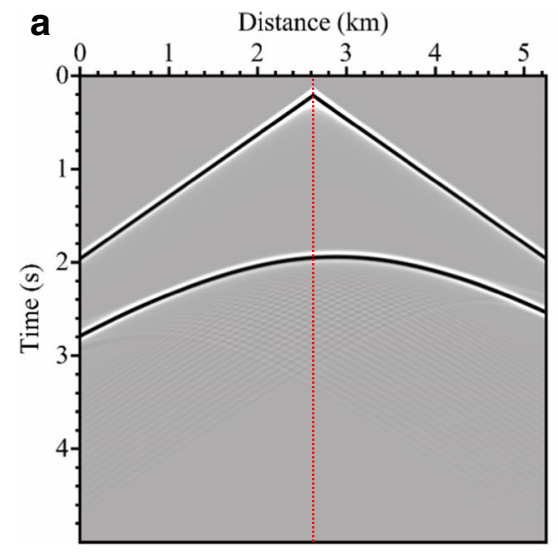

Fig. 9 Synthetic shot gathers produced using the a IGM and b FGM methods. The source is located at the center of the model's top surface and uses a $10-\mathrm{Hz}$ Ricker as the wavelet. The staircase seabed of the IGM yields artificial diffractions with a hyperbolic moveout in

The reason for the FGM method breaking down when interfaces are dipping steeply is because we only interpolate between grid points in the vertical direction. This means that when the dip angle is greater than $45^{\circ}$ then the local interface will lie across more than one cell in depth for each horizontal grid interval, such that steps along the interface are necessary to account for this mismatch when discretizing the model. Fortunately, to minimize numerical dispersion during finite difference, the grid interval is always much smaller than the shortest wavelength-this helps to relax the threshold on interface dip angle. For

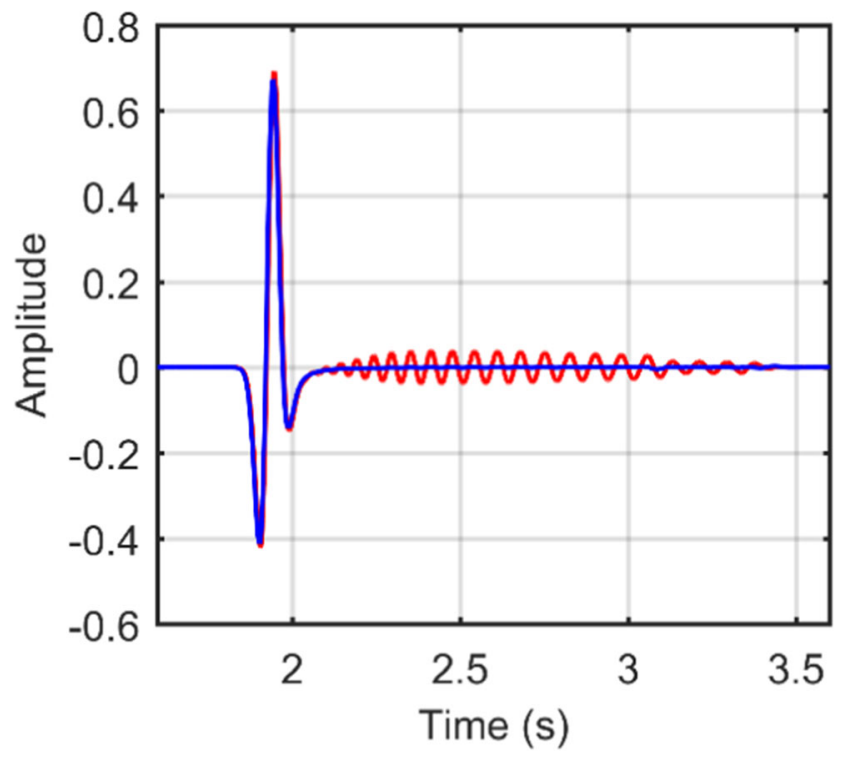

Fig. 10 Comparison between the central trace of each shot record in Fig. 9, where the red trace corresponds to the IGM and the blue to FGM. The red trace can be seen to contain strong oscillations following the main reflection event, which are produced by the inaccurate staircase seabed in the IGM scattering the wavefield

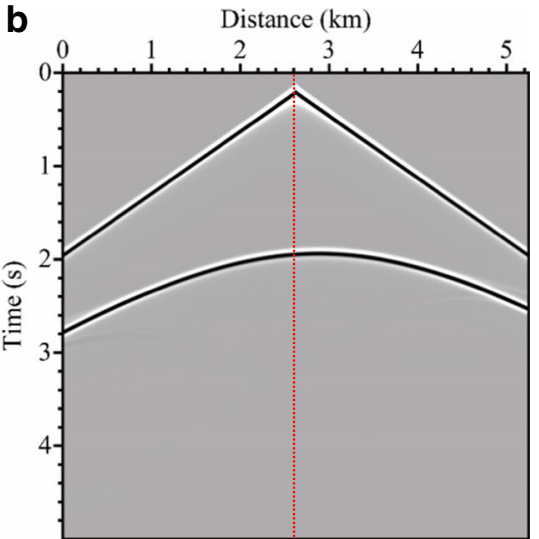

(a). These artificial diffractions are removed in (b) by using fractional grids to shift the seabed interface to its correct position. The red dotted lines are at a horizontal distance of $2625 \mathrm{~m}$ and indicate the position of traces plotted in Fig. 10

instance, the shortest wavelengths in this example are sampled eight and four times when using the $10-$ and $20-\mathrm{Hz}$ Ricker wavelets, respectively. The limit of interface dip angle for diffraction generation for these two cases can be seen in Fig. 12d, which plots the recorded diffraction energy normalized by the recorded wavefield energy of the trace from the zero-dip model. This measurement demonstrates that the spurious diffractions are insignificant up until the interface dips at $76^{\circ}$ or $63^{\circ}$ (for the 10 - and $20-\mathrm{Hz}$ wavelet cases, respectively). It is important to note, on the other hand, that when using the IGM method, conspicuous diffractions are observed in any instance where the interface is not horizontal. With this in mind, the FGM method using interpolation solely in the vertical direction will account for the vast majority of seabed configurations. In the occasion of seabed sections containing extreme dips, the FGM method can instead be applied using interpolation in the horizontal direction.

\subsection{A synthetic seabed model}

The fourth example applies the FGM method to the synthetic seabed model in Fig. 13. The seabed in this model, the location of which is indicated by the red line in Fig. 13, is designed to be somewhat realistic by undulating with lateral position. The velocities $v(x, z)$ of the model's seawater and sedimentary rock are determined using

$v(z)=\left\{\begin{array}{ll}1500 & z<f(x) \\ 0.13 z+1500 & z \geq f(x)\end{array}\right.$,

where $z$ is depth, $x$ is horizontal distance, and $f(x)$ is the position function of the seabed interface. We here take into consideration the variation in density between water and rock. 
Fig. 11 Wavefield snapshot at $1.5 \mathrm{~s}$ when using a the IGM and b the FGM
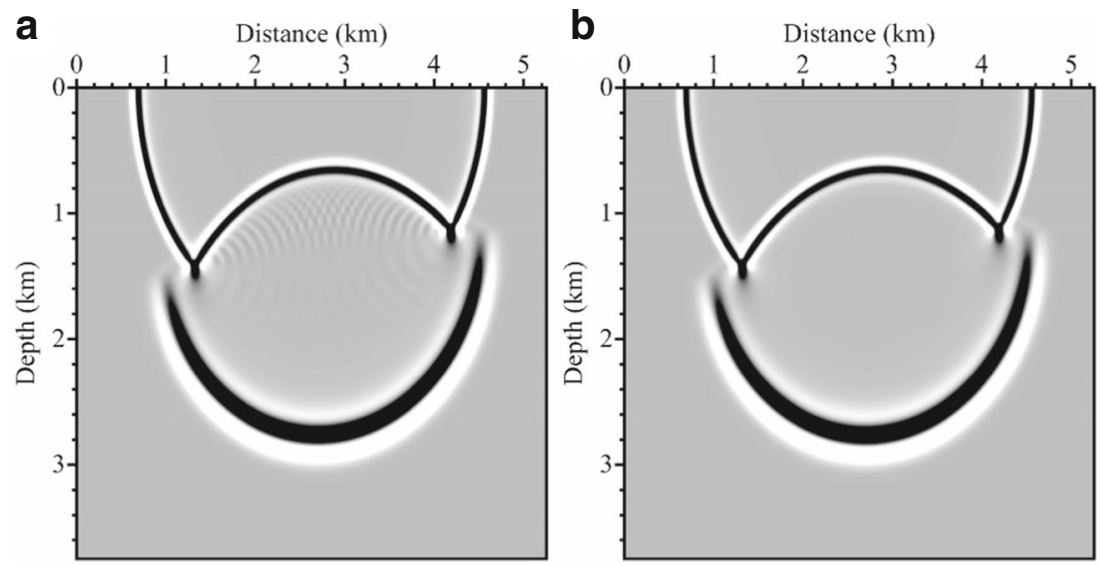

\section{a}

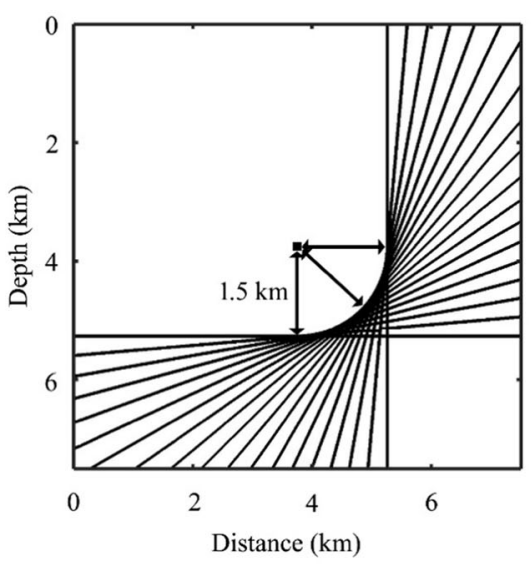

C

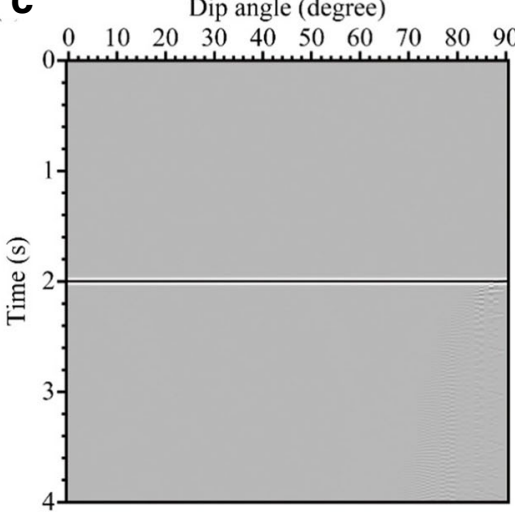

Fig. 12 Analysis of the FGM method's effectiveness at avoiding artificial diffractions for different interface dip angles. a Schematic illustration of the two-layer acoustic velocity model containing different angles of interface dip. The dip angle of the interface changes from $0^{\circ}$ to $90^{\circ}$ over an interval of $1^{\circ}$. The velocities above and below the interface are 1500 and $1800 \mathrm{~m} / \mathrm{s}$, respectively. The densities above and below the interface are 1000 and $1200 \mathrm{~kg} / \mathrm{m}^{3}$, respectively. The source and receiver are located at the center of the model and are always 1.5 $\mathrm{km}$ away from the interface at its closest point. b A synthetic commonreceiver gather for the 91 velocity models, recorded using a $10-\mathrm{Hz}$ b Dip angle (degree)

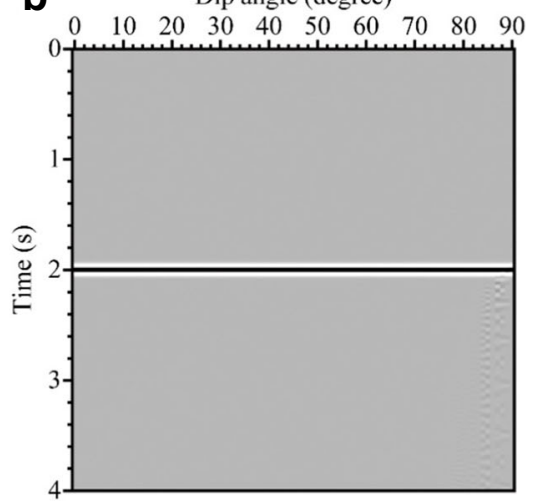

d

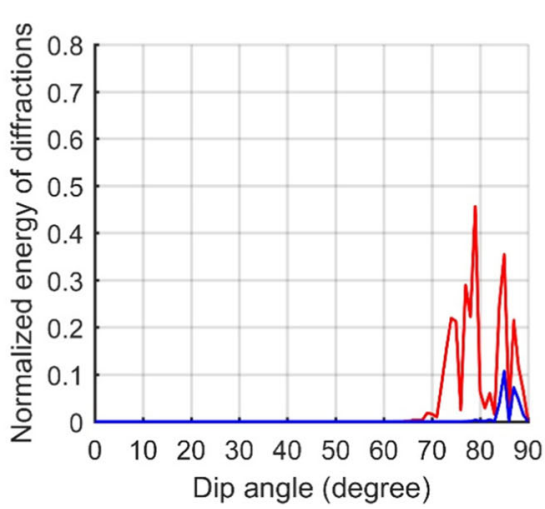

Ricker source wavelet; c the same as (b) but recorded using a $20-\mathrm{Hz}$ Ricker source wavelet. In both cases, the direct arrival has been subtracted. $\mathbf{d}$ Recorded diffraction energy normalized by the energy of the trace from the zero-dip model, which contains no spurious arrivals: the blue and red curves represent the data recorded using the 10- and 20$\mathrm{Hz}$ Ricker wavelets, respectively. The normalized energy is a useful indicator for detecting the presence of artificial features in the wavefield; here, it is lower than $2.5 \mathrm{e}^{-6}$ for the $10-\mathrm{Hz}$ data when the dip angle is less than $76^{\circ}$, and lower than $1 \mathrm{e}^{-4}$ for the $20-\mathrm{Hz}$ data when the angle is smaller than $63^{\circ}$ 
Fig. 13 An acoustic model containing an undulating seabed, sampled using the IGM and FGM methods. a, d The velocity and density models, respectively. c-f Enlarged views of the areas outlined by the gray squares in (a) and (b). c, d The velocity and density models using the FGM method, respectively. $\mathbf{d}, \mathbf{f}$ The counterparts of (c) and (d) by using the IGM method. The red curve in each panel indicates the true position of the seabed

Fig. 14 Comparison between vertical profiles of $\mathbf{a}, \mathbf{c}$ velocity and $\mathbf{b}, \mathbf{d}$ density taken from the models generated using the IGM and FGM methods of Fig. 13. The profiles are across the seabed and taken at horizontal distances of $\mathbf{a}, \mathbf{b} 12.36$ and $\mathbf{c}, \mathbf{d}$ $12.375 \mathrm{~km}$. The blue circles and red crosses correspond to the FGM and IGM methods, respectively, while the black line represents the model prior to sampling

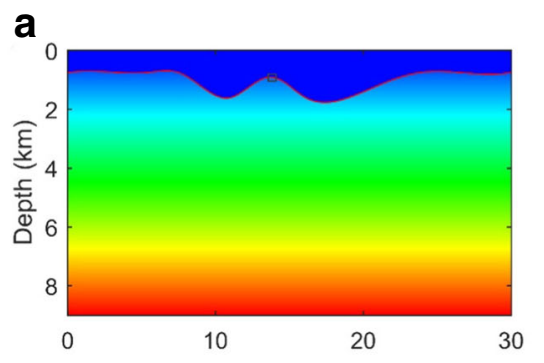

b
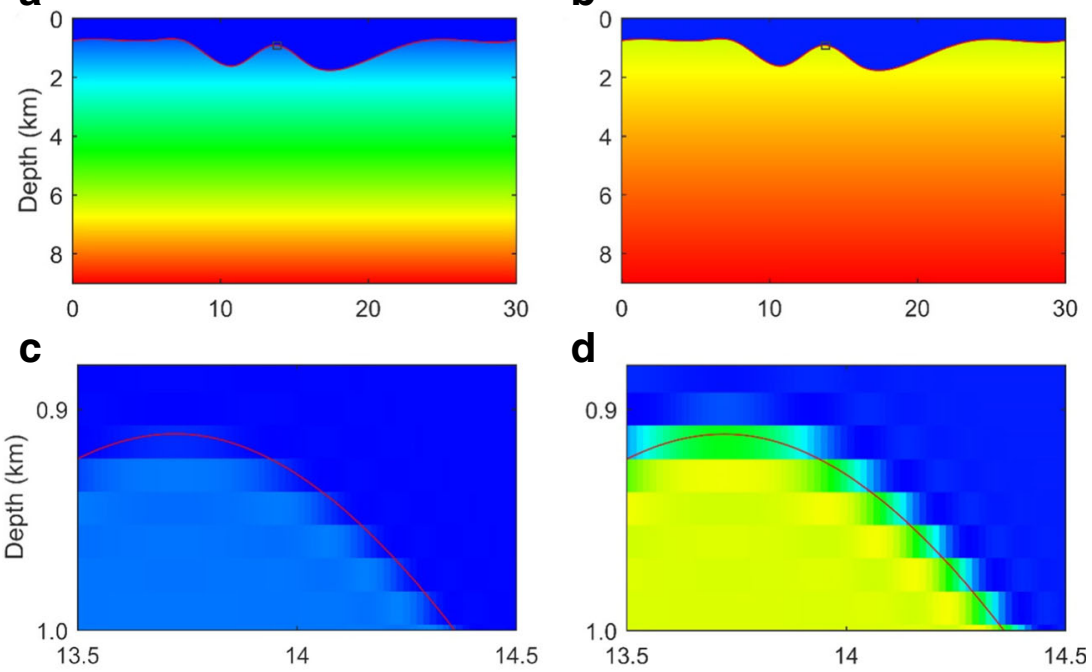

d
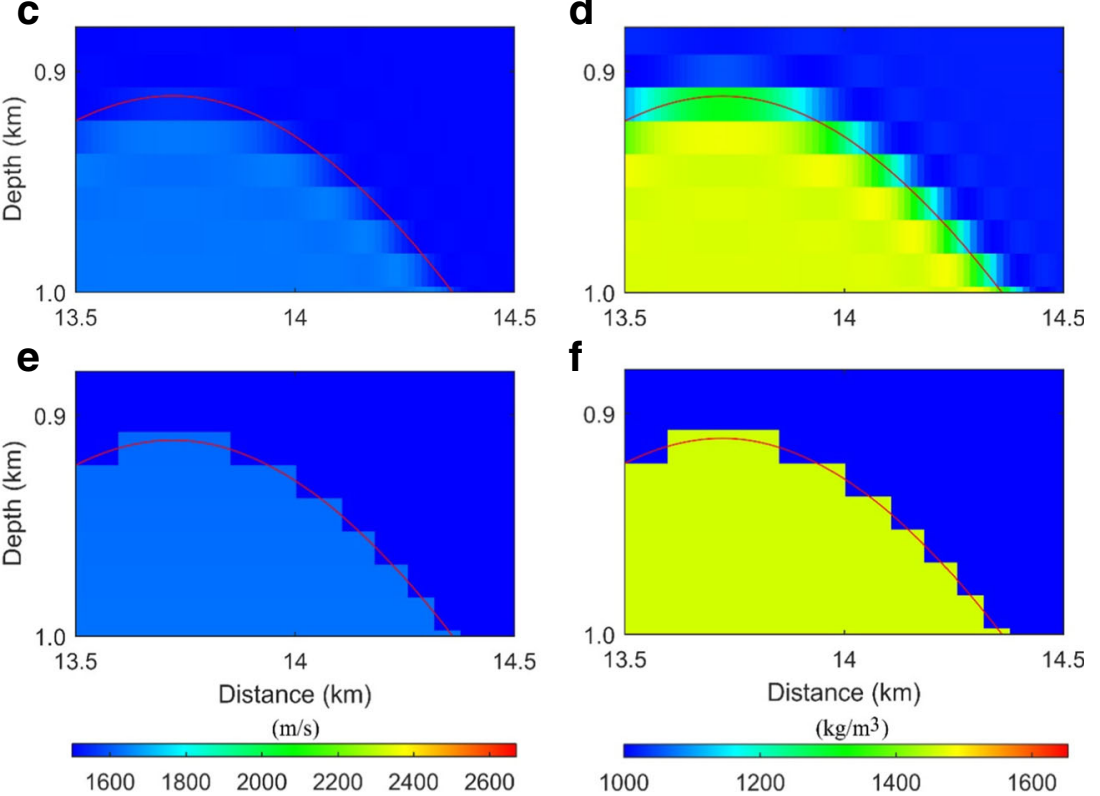

f

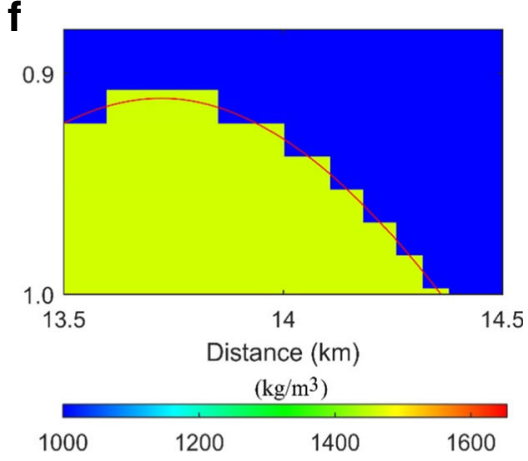

a

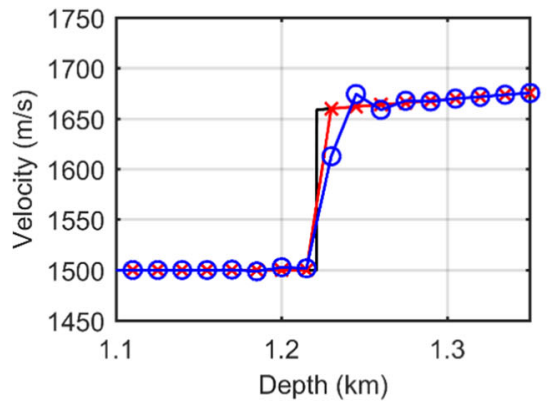

C

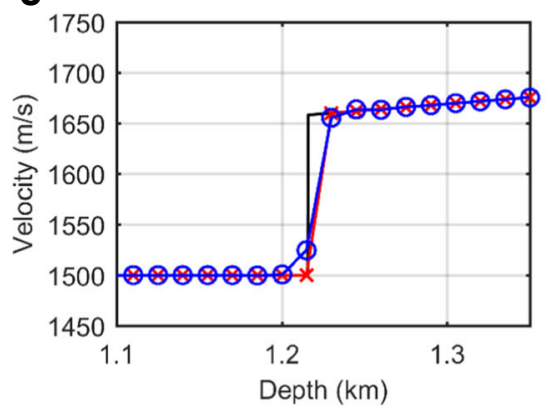

b

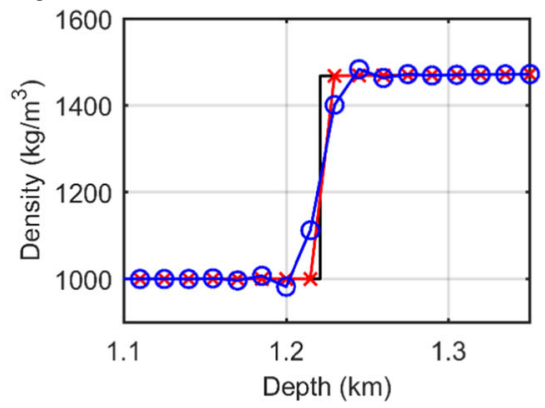

d

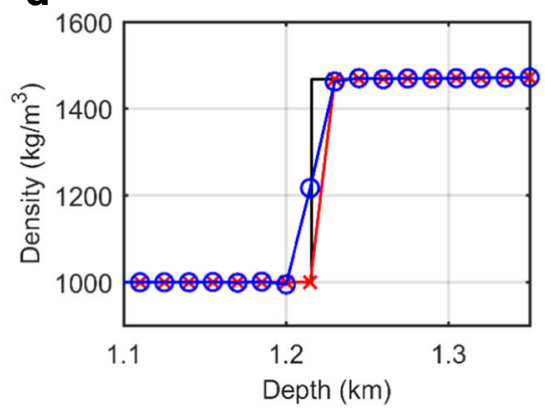


Fig. 15 Shot records generated using a the IGM (Fig. 13a) and b the FGM (Fig. 13b). The red dashed lines in (a) indicate 1.95 $\mathrm{km}$; traces from these positions are compared in Fig. 16. The display is clipped to $5 \%$ of the maximum amplitude of the reflection

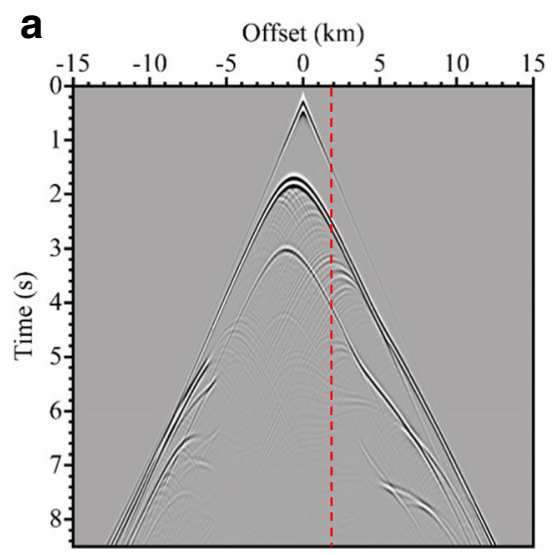

b

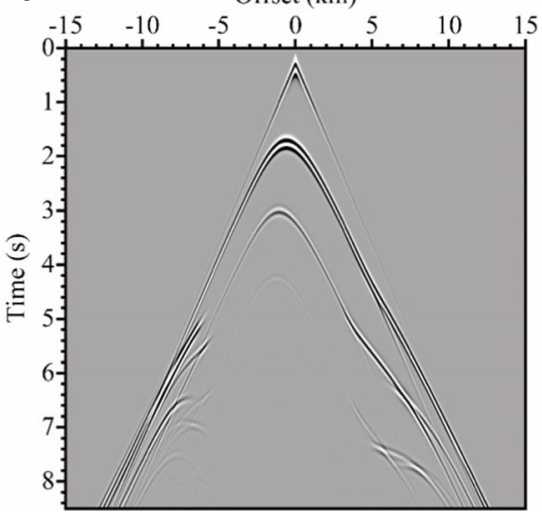

The seawater density is taken to be $1000 \mathrm{~kg} / \mathrm{m}^{3}$, while the rock density is defined using Gardener's relation [14]:

$\rho=0.23 v^{0.25}$,

where $v$ is the rock velocity.

To obtain the IGM model, we sample the model from the top surface (i.e., $z=0 \mathrm{~m}$ ) every $15 \mathrm{~m}$ both vertically and horizontally. To generate the FGM model, the seabed transition point is aligned on the interface defined by $f(x)$ with the average velocity and density of the overlying water and sub-seabed medium computed by using Eqs. 6 and 7. The enlarged view of the seabed of the discrete velocity models with the IGM and FGM methods are shown in Fig. 13c, e, respectively. Figure 13d, f show the density counterparts. The IGM model can be seen to contain a staircased interface at the seabed, where it is apparent that this interface deviates in places from the true seabed position as indicated by the red curve in Fig. 13. By comparison, the model obtained using the FGM method contains a smoother change in both velocity and density at the seabed; this appearance is a result of the vertical interpolation from the fractional grid adopted.
Fig. 16 Shot records generated by using IGMs with the grid size of a $7.5, \mathbf{b} 3.75$, c 1.875, and d $0.9375 \mathrm{~m}$. The display is clipped to the same as that in Fig. 15 a

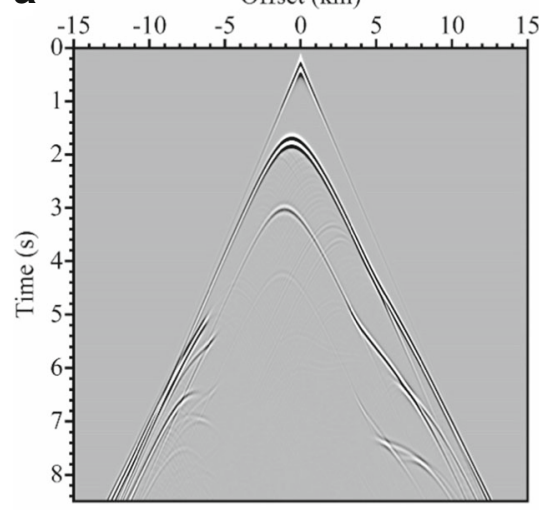

C

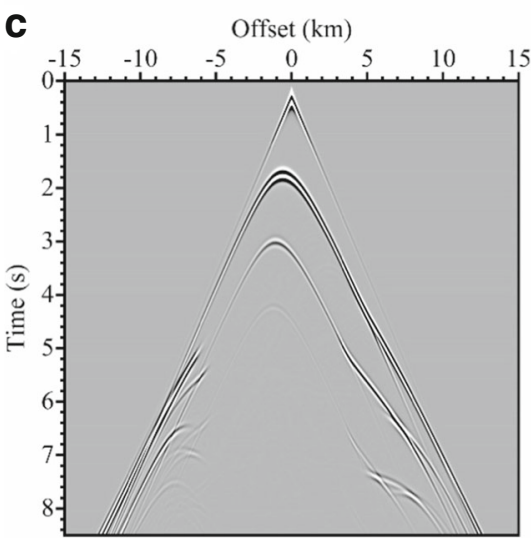

b

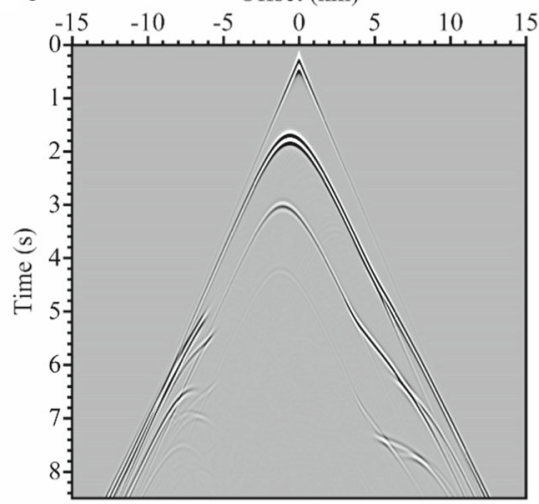

d

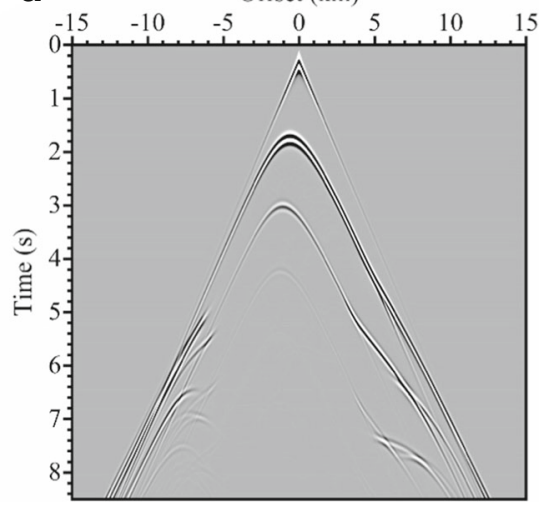




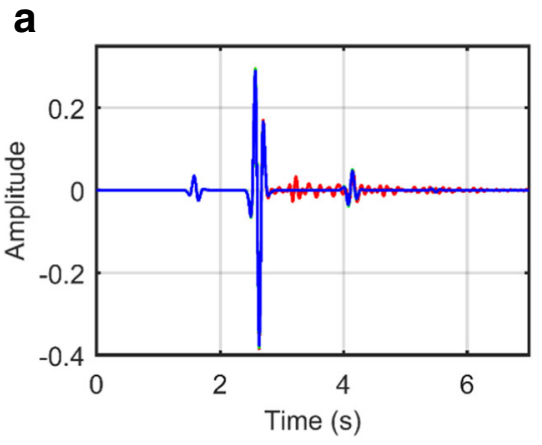

Fig. 17 Comparison between traces from the synthetic shot gathers of Figs. 15 and 16 at offsets of $1.95 \mathrm{~km}$, where these positions are indicated by the red dashed lines in Fig. 15a. b An enlarged view of (a). The red and blue curves represent the records produced using the IGM and FGM, respectively. The green curve almost overlapped by the blue

Figure 14 compares vertical velocity and density profiles at two locations from each of the FGM (blue) and IGM (red) models, as well as the unsampled model (black). Of note in this figure is that, in both instances, the FGM profiles intersect the homogenized velocity and density transition of the true seabed. This is equivalent to having a grid point in each column of the model positioned at the center of the seabed interface, and thus the FGM will result in an accurate representation of the seabed. The IGM profiles, on the other hand, can be seen to intersect the seabed velocity and density transition at incorrect positions.

Figure $15 \mathrm{a}, \mathrm{b}$ show two synthetic shot gathers generated from the IGM and FGM, respectively. The source is located $15 \mathrm{~m}$ below the sea surface and at the center of the model horizontally, while the receivers are fixed at the same depth as the source and spread $15 \mathrm{~km}$ apart across the whole model. The source signal is a Ricker wavelet with a central frequency of $5 \mathrm{~Hz}$. The record generated with the IGM can be seen to contain a series of diffractions (Fig. 15a); these are of a spurious nature, as they are a product of the staircase approximation to the seabed interface in the IGM. On the other hand, the record generated with the FGM is free of any undesired diffraction events, containing only true physical events (direct arrival, primary reflections, multiple reflections, etc.). The high quality of the record from the FGM can be verified by comparing with the records (Fig. 16) from the IGMs with a series of finer grid size, i.e., 7.5, 3.75, 1.875, and $0.9375 \mathrm{~m}$, in both horizontal and vertical directions. As can be seen from Fig. 16, with the decrease of the grid size, the spurious diffractions become gradually weaker. These spurious diffrations are almost inexistent in the example with the grid spacing of $0.9375 \mathrm{~m}$ (Fig. 16d). This demonstrates that using IGM increases the computational cost by a factor of $\left(16^{3}=\right) 4096$ times and the memory cost of $\left(16^{2}\right.$ $=) 256$ times that of the FGM in order to suppress the spurious diffractions and obtain a similar quality in the generated record (Fig. 15b). b

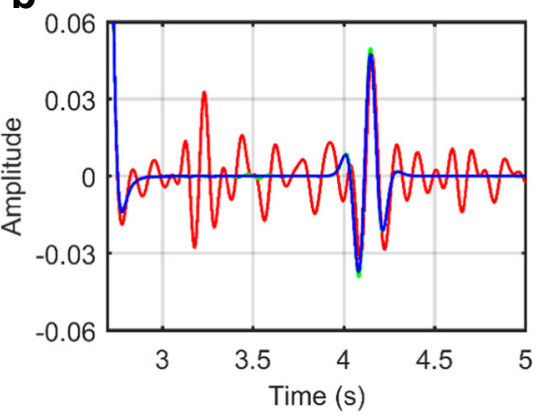

curve indicates the records from the IGM with a grid size of 0.9375 $\mathrm{m}$, which is 16 times finer than the model of FGM. It is clear that the FGM traces are noticeably cleaner, as the IGM traces (the red curves) contain significant amounts of diffraction energy

The higher quality of the records generated by the FGM also can be observed by comparing the traces in Fig. 17. The data from the IGM (red curves) contains strong spurious diffractions after the primary reflections. The spurious diffractions are even faintly noticeable around $3.5 \mathrm{~s}$ in the data from the IGM with a grid size of $0.9375 \mathrm{~m}$ (green curves). On the contrary, the data from the FGM (blue curves) is free of them. However, it can be observed that the FGM with a grid size of $15 \mathrm{~m}$ gives a slightly smaller reflection amplitude (around 3\% in this case) than that of

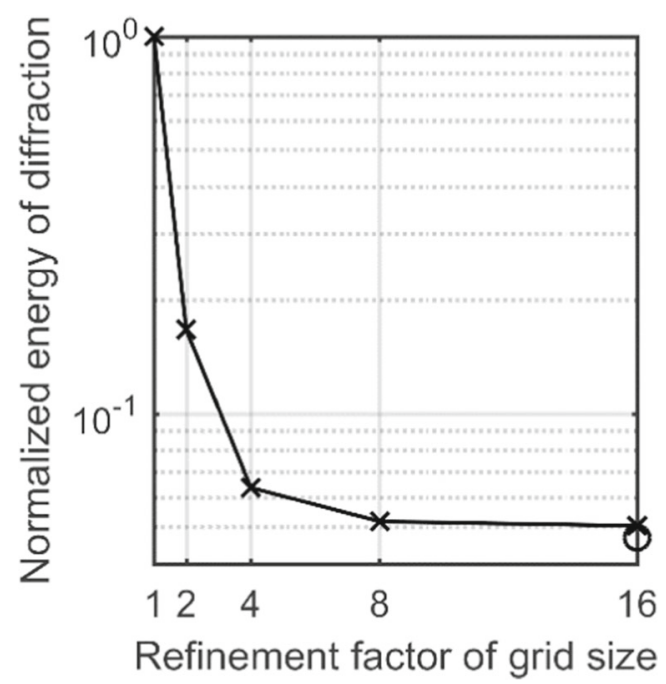

Fig. 18 Normalized energy of the artificial diffractions in the records (Figs 15 and 16) from the models by refining the grid size with a factor of $1,2,4,8$, and 16, which are corresponding to the grid size of 15 , $7.5,3.75,1.875$, and $0.9375 \mathrm{~m}$, respectively. The diffraction energy is the square of the amplitude of the artificial diffractions and then normalized by the diffraction energy of the grid size of $15 \mathrm{~m}$. The artificial diffractions are selected by applying the same mask on all the records to remove the direct arrivals and reflections. The circle indicates the artificial diffraction energy from FGM with a grid size of $15 \mathrm{~m}$. Note that the vertical axis is displayed on a log scale 
the IGM with a grid size of $0.9375 \mathrm{~m}$. This is caused by setting an average value to the grid points at the interface with the homogenization theory, which reduces the sharpness of the discontinuous interface and results in a slightly weaker reflectivity; however, this level of error in the amplitude is very small and more importantly the computed travel time is not affected. This is an especially important aspect as the travel time has the key role in seismic imaging and velocity model building, and for this reason its accuracy is paramount.

To measure the energy of spurious diffractions in the shot gather quantitatively, we masked out the reflections and the direct arrivals. Figure 18 shows the results of this comparison on the energy of spurious diffractions. As expected, the spurious diffraction energy decays exponentially, with decreasing grid spacing. In fact, the FGM gives even slightly smaller diffraction energy than the IGM with a grid size of $0.9375 \mathrm{~m}$.

\section{Discussion}

This work demonstrates the effectiveness of the FGM method when the objective is accurate modeling of seabed bathymetry. This method is not limited to this purpose, however, and can be applied to other scenarios where sharp model interfaces exist, for instance, the transitions from sediments to salt, chalk, and basalt.

Currently, the FGM method works well for the interfaces gradually varying in one direction, which can be the horizontal or vertical direction. However, if the interfaces have drastic changes along all directions, the FGM method may be unable to improve the quality of modeling compared with the IGM method.

For the scenario of multi-interfaces, we can apply the FGM method to interfaces one by one and then combine them together to form the final FGM. However, if any two interfaces have a distance less than the half window of the Kaiser function, e.g., eight grid points in this paper, the combination of models for each individual interfaces may cancel out the useful oscillation near the interfaces and results in compromised modeling.

In the case of a model that has already been discretized with the IGM method, we still could convert it into an FGM, if the precise position of the interfaces is known. However, if the position of the interfaces is unknown, the position has to be inferred before the conversion.

\section{Conclusions}

The accurate representation of the seabed in models used for finite difference modeling is often challenging, as its bathymetry in cases can be highly variable and more often than not it results in a strong contrast in physical properties.

The traditional method for model discretizationreferred to as the integer grid model (IGM) methodinvolves sampling from the top surface over a fixed grid interval. The IGM technique, due to its simple nature, can result in the misrepresentation of interfaces in the original model, particularly if their shape is irregular. This will cause finite difference schemes to produce inaccurate travel times for seabed reflection events, as well as, artificial diffractions that can contaminate other physical events. To circumvent these problems, we have introduced a novel method, based on the concept of fractional grid models (FGMs), which generates models for finite difference schemes that are discretized accurately. This is achieved by ensuring that the discretized seabed interface intersects the average velocity and density of the known seabed, such that it is therefore aligned with the seabed's actual position. Models generated using the FGM method are compatible with existing finite-difference algorithms and do not entail a significant computational cost, as they do not require any compromise in terms of grid spacing nor time-stepping interval. We have presented four numerical examples that demonstrate how the FGM method produces correct travel times and avoids the artificial diffractions associated with the IGM method.

Acknowledgments The authors would like to thank the editor's and two anonymous reviewers' very useful suggestions and comments to complete the literature reviews and the theory of this presented method.

Funding Information This work was partially supported by the NSFC (Grant No. 41504106) State Key Laboratory of Petroleum Resource and Prospecting Grant (No. PRP/indep-3-1508), and Science Foundation of China University of petroleum, Beijing (No. 2462015YJRC012).

Open Access This article is distributed under the terms of the Creative Commons Attribution 4.0 International License (http:// creativecommons.org/licenses/by/4.0/), which permits unrestricted use, distribution, and reproduction in any medium, provided you give appropriate credit to the original author(s) and the source, provide a link to the Creative Commons license, and indicate if changes were made.

\section{References}

1. Alford, R.M., Kelly, K.R., Boore, D.M.: Accuracy of finitedifference modeling of the acoustic wave equation. Geophysics 39(5), 834-842 (1974). https://doi.org/10.1190/1.1440470

2. Backus, G.E.: Long-wave elastic anisotropy produced by horizontal layering. J. Geophys. Res. 67(11), 4427-4440 (1962)

3. Baysal, E., Kosloff, D.D., Sherwood, J.W.C.: Reverse time migration. Geophysics 48(11), 1514-1524 (1983). https://doi.org/10.1190/1.1441434 
4. Berkhout, A.J.: Seismic Migration Imaging of Acoustic Energy by Wave Field Extrapolation. A. Theoretical aspects. Elsevier, Amsterdam (1982)

5. Brown, D.: A note on the numerical solution of the wave equation with piecewise smooth coefficients. Math. Comput. 42(166), 369391 (1984). https://doi.org/10.2307/2007591

6. Chung, E.T., Engquist, B.: Optimal discontinuous Galerkin methods for wave propagation. SIAM J. Numer. Anal. 44(4), 21312158 (2006). https://doi.org/10.1137/050641193

7. Chung, E.T., Engquist, B.: Optimal discontinuous Galerkin methods for the acoustic wave equation in higher dimensions. SIAM J. Numer. Anal. 47(4), 3820-3848 (2009)

8. Cohen, G., Fauqueux, S.: Mixed finite elements with masslumping for the transient wave equation. J. Comput. Acoust. 8(1), 171-188 (2000). https://doi.org/10.1142/S0218396X00000 $11 \mathrm{X}$

9. Chu, C., Stoffa, P.: Determination of finite-difference weights using scaled binomial windows. Geophysics 77(3), W17-W26 (2012). https://doi.org/10.1190/geo2011-0336.1

10. Dumbser, M., Käser, M., de la Puente, J.: Arbitrary high-order finite volume schemes for seismic wave propagation on unstructured meshes in 2D and 3D. Geophys. J. Int. 171(2), 665-694 (2007). https://doi.org/10.1111/j.1365-246X.2007.03421.x

11. Etienne, V., Chaljub, E., Virieux, J., Glinsky, N.: An hpadaptive discontinuous Galerkin finite-element method for 3D elastic wave modeling. Geophys. J. Int. 183(2), 941-962 (2010). https://doi.org/10.1111/j.1365-246X.2010.04764.x

12. Fogarty, T.R., LeVeque, R.J.: High-resolution finite-volume methods for acoustic waves in periodic and random media. J. Acoust. Soc. Amer. 106(1), 17-28 (1999)

13. Fornberg, B.: The pseudospectral method: accurate representation of interfaces in elastic wave calculations. Geophysics 53(4), 625637 (1988). https://doi.org/10.1190/1.1442497

14. Gardner, G., Gardner, L., Gregory, A.: Formation velocity and density - the diagnostic basics for stratigraphic traps. Geophysics 39(5), 770-780 (1974). https://doi.org/10.1190/1.1440465

15. He, X., Yang, D., Wu, H.: A weighted Runge-Kutta discontinuous Galerkin method for wavefield modeling. Geophys. J. Int. 200(3), 1389-1410 (2015). https://doi.org/10.1093/gji/ggu487

16. Hestholm, S., Ruud, B.: 2D finite-difference elastic wave modeling including surface topography. Geophys. Prospect. 42(4), 371390 (1994). https://doi.org/10.1111/j.1365-2478.1994.tb00216.x

17. Hestholm, S., Ruud, B.: 3D finite difference elastic wave modeling including surface topography. Geophysics 63(2), 613-622 (1998). https://doi.org/10.1190/1.1444360

18. Hicks, G.: Arbitrary source and receiver positioning in finitedifference schemes using Kaiser windowed sinc functions. Geophysics 67(1), 156-165 (2002). https://doi.org/10.1190/1.1451454

19. Hu, W.: An improved immersed boundary finite-difference method for seismic wave propagation modeling with arbitrary surface topography. Geophysics 81(5), T311-T322 (2016). https://doi.org/10.1190/geo2016-0094.1

20. Kaiser, J.K.: Nonrecursive digital filter design using the I0-sinh window function. In: Proceedings of the International Symposium on Circuits and Systems, pp. 20-23. IEEE (1974). Reprinted in Oppenheim, A. V. (ed.), Selected papers in digital signal processing, II. IEEE Press, 123-126 (1976)

21. Käser, M., Dumbser, M.: An arbitrary high-order discontinuous Galerkin method for elastic waves on unstructured meshes-I. The two-dimensional isotropic case with external source terms. Geophys. J. Int. 166(2), 855-877 (2006). https://doi.org/10.1111/j.1365-246X.2006.03051.x

22. Komatitsch, D., Coute, F., Mora, P.: Tensorial formulation of the wave equation for modeling curved interfaces. Geophys. J. Int.
127(1), 156-168 (1996). https://doi.org/10.1111/j.1365-246X.1996. tb01541.x

23. Komatitsch, D., Tsuboi, S., Tromp, J.: The spectral-element method in seismology. In: Seismic Earth: Array Analysis of Broadband Seismograms, pp. 205-227. American Geophysical Union (2013). https://doi.org/10.1029/157GM13

24. Komatitsch, D., Vilotte, J.-P.: The spectral element method: an efficient tool to simulate the seismic response of 2D and 3D geological structures. B. Seismol. Soc. Am. 88(2), 368-392 (1998)

25. Levander, A.R.: Fourth-order finite-difference P-SV seismograms. Geophysics 53(11), 1425-1436 (1988). https://doi.org/10. $1190 / 1.1442422$

26. LeVeque, R., Li, Z.: The immersed interface method for elliptic equations with discontinuous coefficients and singular sources. SIAM J. Numer. Anal. 31(4), 1019-1044 (1994)

27. Levin, S.A.: Principle of reverse-time migration. Geophysics 49(4), 581-583 (1984). https://doi.org/10.1190/1.1441693

28. Liu, Y.: Globally optimal finite-difference schemes based on least squares. Geophysics 78(4), T113-T132 (2013). https://doi.org/10.1190/geo2012-0480.1

29. Lombard, B., Piraux, J., Gelis, C., Virieux, J.: Free and smooth boundaries in 2-D finite-difference schemes for transient elastic waves. Geophys. J. Int. 172(1), 252-261 (2008). https://doi.org/10.1111/j.1365-246X.2007.03620.x

30. Lysmer, J., Drake, L.A.: A finite element method for seismology. Methods Comput. Phys. 11, 181-216 (1972)

31. Mansur, W.J., Brebbia, C.A.: Numerical implementation of the boundary elementmethod for two-dimensional transient scalar wave propagation problems. Appl. Math. Model 6(4), 299-306 (1982). https://doi.org/10.1016/S0307-904X(82)80038-3

32. Mansur, W.J., Brebbia, C.A.: Formulation of the boundary element method for transient problems governed by the scalar wave equation. Appl. Math. Model. 6(4), 307-311 (1982). https://doi.org/10.1016/S0307-904X(82)80039-5

33. McMechan, G.A.: Migration by extrapolation of time-dependent boundary values. Geophys. Prospect. 31(3), 413-420 (1983). https://doi.org/10.1111/j.1365-2478.1983.tb01060.x

34. Muir, F., Dellinger, J., Etgen, J., Nichols, D.: Modeling elastic fields across irregular boundaries. Geophysics 57(9), 1189-1193 (1992)

35. Mulder, W.A.: Higher-ordermass-lumped finite elements for the wave equation. J. Comput. Acoust. 9(2), 671-680 (2001). https://doi.org/10.1142/S0218396X0100067X

36. Pratt, R.G.: Seismic waveform inversion in the frequency domain. Part 1: Theory and verification in a physical scale model. Geophysics 64(3), 888-901 (1999). https://doi.org/10.1190/1.1444597

37. Pratt, R.G., Shipp, R.M.: Seismic waveform inversion in the frequency domain. Part 2: Fault delineation in sediments using crosshole data. Geophysics 64(3), 902-914 (1999). https://doi.org/10.1190/1.1444598

38. Rao, Y., Wang, Y.: Seismic waveform simulation with pseudoorthogonal grids for irregular topographic models. Geophys. J. Int. 194(3), 1778-1788 (2013). https://doi.org/10.1093/gji/ggt190

39. Robertsson, J.O.A.: A numerical free-surface condition for elastic/viscoelastic finite-difference modeling in the presence of topography. Geophysics 61(5), 1921-1934 (1996). https://doi.org/10.1190/1.1444107

40. Schoenberg, M., Muir, F.: A calculus for finely layered anisotropic media. Geophysics 54(4), 581-589 (1989)

41. Symes, W., Terentyev, I.: Subgrid modeling via mass lumping in constant density acoustics. In: SEG Technical Program Expanded Abstracts 2009, pp. 2572-2576 (2009)

42. Symes, W.W., Vdovina, T.: Interface error analysis for numerical wave propagation. Comput. Geosci. 13(3), 363-371 (2009). https://doi.org/10.1007/s10596-008-9124-8 
43. Tarantola, A.: Inversion of seismic reflection data in the acoustic approximation. Geophysics 49(7), 1259-1266 (1984). https://doi.org/10.1190/1.1441754

44. Virieux, J.: SH-wave propagation in heterogeneous media: velocity-stress finite-difference method. Geophysics 49(11), 1933-1942 (1984). https://doi.org/10.1190/1.1441605

45. Virieux, J.: P-SV wave propagation in heterogeneous media: velocity-stress finite-difference method. Geophysics 51(4), 889901 (1986). https://doi.org/10.1190/1.1442147

46. Vossen, R.v., Robertsson, J.O.A., Chapman, C.H.: Finite-difference modeling of wave propagation in a fluidsolid configuration. Geophysics 67(2), 618-624 (2002). https://doi.org/10.1190/1.1468623

47. Wang, Y., Liang, W., Nashed, Z., Li, X., Liang, G., Yang, C.: Seismic modeling by optimizing regularized staggered-grid finite-difference operators using a time-space-domain dispersionrelationship-preserving method. Geophysics 79(4), T277-T285 (2014). https://doi.org/10.1190/geo2014-0078.1

48. Warner, M. et al.: Anisotropic 3D full-waveform inversion. Geophysics 78(2), R59-R80 (2013). https://doi.org/10.1190/geo20120338.1

49. Whitmore, N.D.: Iterative depth migration by backward time propagation. In: SEG Technical Program Expanded Abstracts 1983, pp. 382-385 (1983). https://doi.org/10.1190/1.1893867

50. Wu, D., Yao, G., Cao, J., Wang, Y.: Least-squares RTM with L1 norm regularisation. J. Geophys. Eng. 13(4), 666-673 (2016). https://doi.org/10.1088/1742-2132/13/5/666

51. Yao, G., Wu, D., Debens, H.A.: Adaptive finite difference for seismic wavefield modeling in acoustic media. Sci. Rep. 6, 30302 (2016). https://doi.org/10.1038/srep30302
52. Yao, G., Jakubowicz, H.: Least-squares reverse-time migration in a matrix-based formulation. Geophys. Prospect. 64(3), 611-621 (2016). https://doi.org/10.1111/1365-2478.12305

53. Yao, G., Wu, D.: 2015. Least-squares reverse-time migration for reflectivity imaging. Sci. China Earth Sci. 58(11), 1982-1992 (2015). https://doi.org/10.1007/s11430-015-5143-1

54. Zhang C., Symes W.W.: Fourth order, full-stencil immersed interface method for elastic waves with discontinuous coefficients. In: SEG Technical Program Expanded Abstracts 1998, pp. 19291932 (1998). https://doi.org/10.1190/1.1820315

55. Zhang, W., Chen, X.: Traction image method for irregular free surface boundaries in finite difference seismic wave simulation. Geophys. J. Int. 167(1), 337-353 (2006). https://doi.org/10.1111/j.1365-246X.2006.03113.x

56. Zhang, C., LeVeque, R.J.: The immersed interface method for acoustic wave equations with discontinuous coefficients. Wave Motion 25(3), 237-263 (1997). https://doi.org/10.1016/S0165-21 25(97)00046-2

57. Zhang, J., Liu, T.: Elastic wave modeling in 3D heterogeneous media: 3D grid method. Geophys. J. Int. 150(3), 780-799 (2002). https://doi.org/10.1046/j.1365-246X.2002.01743.x

58. Zhang, J., Yao, Z.: Optimized finite-difference operator for broadband seismic wave modeling. Geophysics 78(1), A13-A18 (2012). https://doi.org/10.1190/geo2012-0277.1

59. Zhang, W., Zhuang, Y., Chung, E.T.: A new spectral finite volume method for elastic wave modeling on unstructured meshes. Geophys. J. Int. 206(1), 292-307 (2016). https://doi.org/10.1093/gji/ggw148

60. Zhang, Y., Zhang, H., Zhang, G.: A stable TTI reverse time migration and its implementation. Geophysics 76(3), WA3-WA11 (2011). https://doi.org/10.1190/1.3554411 